\title{
Prediction of Free Surface Roughening by 2D and 3D Model Considering Material Inhomogeneity*
}

\author{
Tsuyoshi FURUSHIMA**, Tetsuro MASUDA**, Ken-ichi MANABE** \\ and Sergei ALEXANDROV*** \\ ${ }^{* *}$ Department of mechanical engineering, Tokyo Metropolitan University \\ 1-1 Minami-ohsawa, Hachioji, Tokyo, Japan \\ E-mail: furushima-tsuyoshi@tmu.ac.jp \\ ***Institute for Problems in Mechanics, Russian Academy of Sciences, Russia \\ 101-1, Prospect Vernadskogo, Moscow, 119526
}

\begin{abstract}
A Finite element (FE) model considering mesoscopic material inhomogeneity due to a different flow stress for each crystal grain to predict free surface roughening is proposed. The effect of the standard deviation of the material inhomogeneity and grain size was investigated by the 2D FE model. In addition, to verify the model considering material inhomogeneity, the free surface roughening behavior is predicted under a bi-axial tension state using the 3D FE model. Furthermore, by comparison with experimental results, the validity of the FE model considering material inhomogeneity and its determination method is discussed. The standard deviation $\sigma_{\mathrm{sd}}$ of the material inhomogeneity parameter $\alpha$ in the model has a strong correlation with the rate of increase in the surface roughness, which increases with increasing grain size, is agreement with the conventional empirical equation. The surface roughness under bi-axial tension state is larger than that under uni-axial tension state. In addition, the experimental results are in good agreement with the simulation results. From these findings, we can verify the fundamental behavior and the validity of this model considering material inhomogeneity for the prediction of free surface roughening.
\end{abstract}

Key words: Free Surface Roughening, Material Inhomogeneity, FE Model

\section{Introduction}

Recently, there has been increased demand for the miniaturization of parts for technical applications such as electronic devices, medical equipment, sensor technology and optoelectronics. These micro devices are mainly made of silicon or glass, and are fabricated by semiconductor manufacturing methods. However, semiconductor manufacturing methods have many issues such as the limitations of the applicable materials, and the requirement of massive manufacturing devices with high cost and productivity. In scaling down the dimensions of conventional metal forming technology, various factors must be considered. So far, studies on micro forming have mainly been conducted from the viewpoints of materials, working processes, and processing machines ${ }^{(1)-(4)}$.

On the other hand, in the miniaturization of sheet metal forming processes, the ratio of the surface roughness of the material to the total thickness increases with decreasing sheet

*Received 19 July, 2011 (No. 11-0414) [DOI: 10.1299/jmmp.5.978]

Copyright $\odot 2011$ by JSME 
thickness, which must be considered as a non-uniformity of thickness. In particular, the surface roughness increases on the free surface that does not come into contact with the tool. When forming a sheet metal, the free surface of the sheet becomes rough with increasing plastic strain. This is well-known roughening phenomenon on a free surface and has a strong effect not only on the surface quality of a product but also on the forming limit ${ }^{(5)-(7)}$. Therefore, surface roughening is one of the important technical issues in sheet metal forming, particularly in micro materials such as thin sheets and foils used in micro forming. At the micro-scale, the forming limit of thin sheets and foils decreases because the surface roughness and asperities of the micro materials are regarded as variations in the thickness of the micro materials. In other words, the surface roughness gradually increases with increasing plastic strain, and localized necking begins to develop from the deepest troughs of surface asperities.

The roughening phenomenon on the free surface of a polycrystalline metal is closely related to the inhomogeneous deformation of each grain located near the free surface ${ }^{(8)}$. It is known that the inhomogeneous deformation is caused by difference in the crystal orientations of grains at the surface layer. Many experimental studies have been carried out to investigate the effects of the grain size and crystal structure on the roughening phenomenon on a free surface. It was previously reported that the surface roughness on a free surface increases approximately in proportion to the equivalent plastic strain and the grain size as shown by Eq. (1) ${ }^{(5)-(8)}$.

$$
R a=c d \varepsilon_{e q}+R_{0}
$$

here, $R a$ is surface roughness, $c$ is a material constant based on the crystal structure factor, $d$ is average grain size, $\varepsilon_{e q}$ is equivalent plastic strain and $R_{0}$ is initial surface roughness.

However, most of these previous studies dealt with the free surface roughening under simple stress states without contact with tools, such as uniaxial stress and equi-biaxial tension. In actual metal forming, the behavior of free surface roughening is more complex, because contact and non-contact between the material and tools occur several times, making the stress state complicated and varied.

Free surface roughening is caused by material inhomogeneity, such as grain orientation, crystal structure and grain size. Some researchers have modeled material inhomogeneity at the scale of grain size to predict the free surface roughening by finite element (FE) simulation. An advantage of the FE simulation is that it can be applied to the practical metal forming process. To consider material inhomogeneity, Zhao et al. applied a crystal plasticity (CP) FE model ${ }^{(9),(10)}$. In case of CPFE model, the material inhomogeneity can be obtained from the crystal orientation measured with Electron Backscatter Diffraction (EBSD). However, in the CPFE model, the modeling is complex, the convergence performance is poor and the calculation cost is high. Therefore, it is not easy to apply the model to practical metal forming. Additionally, most of the previous studies was focusing on the effect of texture pattern and crystal orientation on free surface roughening under simple stress state. However, the comparison of simulation results obtained from CPFE model with experimental results was not enough.

On the other hand, Yamaguchi ${ }^{(8)}$, Utsunomiya ${ }^{(11),(12)}$ and Furushima ${ }^{(13)}$ proposed a simpler FE model in which the flow stress of each crystal grains in the material is assumed to be different to consider the material inhomogeneity. In their simple FE model considering the flow stress of each crystal grain, a conventional commercial FE code can be easily utilized. In addition, the calculation cost is low and the applicability of the simulation to the practical metal forming process is high, enabling the prediction of free surface roughening using a simple model. In addition, the free surface roughening behavior can be modeled reasonably and easily using the simple model. However, it is not easy to determine the microscopic material inhomogeneity in the case of that the flow stress of crystal grains in a material is different for each grain. In particular, the physical meaning of 
the inputted material inhomogeneity parameter is not known, and its determination is fuzzified. In previous studies, the flow stress for each grain was determined by performing a tensile test on a single crystal, but this method takes a long time to obtain the material inhomogeneity. Furushima also attempted to determine the material inhomogeneity parameter by variation in hardness obtained from micro vicers hardness test ${ }^{13)}$. However, FE analysis using the material inhomogeneity parameter obtained from micro hardness test was not in agreement with experimental results.

With this background, in this study, we proposed an FE model considering microscopic material inhomogeneity due to different flow stresses for each crystal grain to predict free surface roughening in practical applications. Fundamental characteristics of suggested inhomogeneous model can be verified by 2D FE models. The effect of the standard deviation of the material inhomogeneity parameter and grain size was investigated by the 2D FE model. In addition, to verify the model considering material inhomogeneity, the free surface roughening behavior was predicted under bi-axial tension state using the 3D FE model. Furthermore, by comparison with experimental results, the validity of the FE model considering material inhomogeneity and its determination method is discussed.

\section{FE Simulation}

\subsection{FE Model Considering Mesoscopic Material Inhomogeneity}

Generally, a polycrystal metal should be dealt with by considering the microscopic inhomogeneity due to crystal grains and orientation. Free surface roughening is caused by the material inhomogeneity. To simulate the surface roughening behavior, an FE simulation considering material inhomogeneity was performed. As the method used to model the material inhomogeneity, a CPFE model based on microscopic information and deformation behavior such as grain orientation and the sliding of each grain in a single crystal was used. However, in the CPFE model, the modeling is complex, the convergence performance is poor and the calculation cost is high. Meanwhile, consideration of the rotation of the crystal orientation in the microscopic texture is not necessarily required for the prediction of free surface roughening. Thus, in this study, the material inhomogeneity was simplified to that at the mesoscale, between the microscale considered in crystal plasticity theory and the macro scale considered in normal continuum mechanics. To consider the mesoscopic material inhomogeneity, a different flow stress in each crystal grain is considered using a conventional FE model. In this FE model, the crystal grains are assumed to be a square group consisting of elements as shown in Fig. 1. The each grain in the square group has the same material properties of flow stress. In this paper, the flow stress of each grain is treated using the equation.

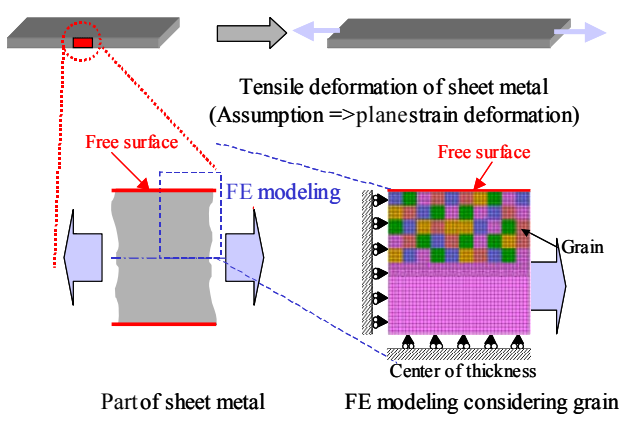

(a) 2D model

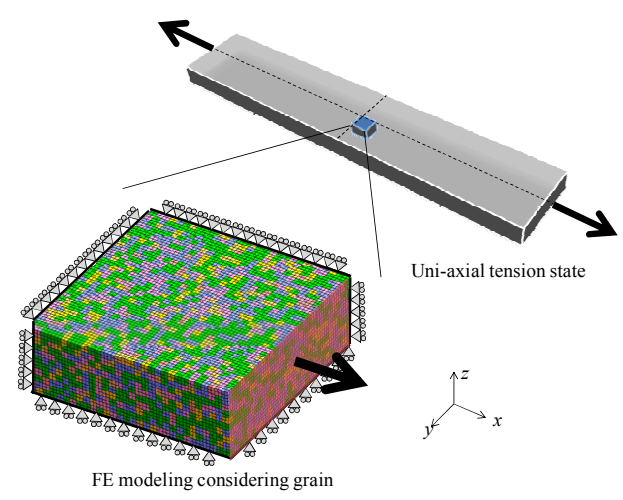

(b) 3D model

Fig. 1 Schematic illustration of 2D and 3D FE models considering material inhomogeneity. 


$$
\sigma=\alpha F \varepsilon^{n}
$$

where $\alpha$ is material inhomogeneity parameter in the model. The probability distribution of the $\alpha$ value in the FE model is divided into $k$ classes as shown in Fig. 2. The probability distribution of the $\alpha$ value used in the model is normal distribution. Thus, the variation in the material inhomogeneity can be expressed by the standard deviation $\sigma_{\text {sd }}$ of the $\alpha$ value. The strain hardening sensitivity index ( $n$-value) of each crystal grain does not vary with the flow stress of a single crystal (3), (5), (6). Additionally, note that crystal rotation and grain sliding are not considered in this model to simplify the problem and prevent the use of excess computational resources.

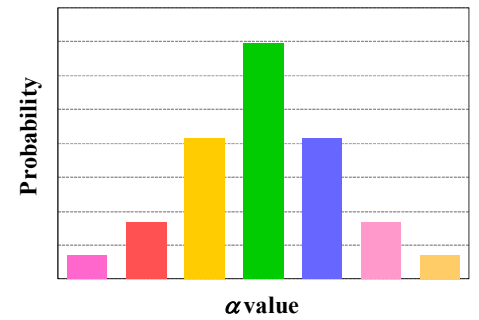

Fig. 2 Schematic illustration of statistical normal distribution of $\alpha$ value.

\subsection{D FE Model}

To investigate the effect of the material inhomogeneity parameter $\alpha$ and grain size, a 2D FE simulation considering material inhomogeneity is performed ${ }^{(14)}$. The roughening of the free surface during plastic deformation is modeled using Marc/Mentat ver.2008. One of the parts of the material is modeled assuming plane strain tensile deformation. In this model, crystal grains are assumed to be square with a side length of $25 \mu \mathrm{m}$ as shown in Fig. 3. Each element in a square group of $4 \times 4$ elements is given the same material properties. The crystal grains are modeled in the neighborhood of the free surface because the internal crystal grains in the material do not influence the roughness behavior on the free surface ${ }^{(8)}$. The depth of the layer below the free surface in which the crystal grains are considered is $125 \mu \mathrm{m}$ in this model. The sheet is deformed at $5 \mu \mathrm{m} / \mathrm{s}$ in the horizontal direction. In this model, three statistical probability distribution of $\alpha$ are defined, which are assumed by arbitrarily changing the crystal orientation to the tensile direction. The three conditions of normal statistical distributions used in the analysis have $\alpha$ values in the range from 0.8 to $1.2\left(\sigma_{\mathrm{sd}}=0.130\right), 0.6$ to $1.4\left(\sigma_{\mathrm{sd}}=0.260\right)$ and 0.4 to $1.6\left(\sigma_{\mathrm{sd}}=0.389\right)$. In these models, the average value of $\alpha$ should be 1.0. This means that these materials of the three conditions are equivalent macroscopically. Figure 4 shows the statistical distributions of $\alpha$ for each crystal grain used in this simulation. Additionally, to investigate the effect of grain size on free surface roughening behavior, three grain size of $25,12.5$ and $6.25 \mu \mathrm{m}$ were used in the simulation as shown in Fig. 5. 


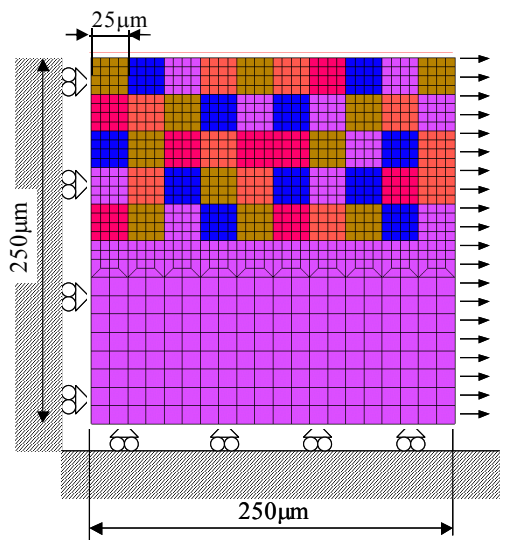

Fig. 3 2D FE model considering material inhomogeneity under plane strain tensile deformation.

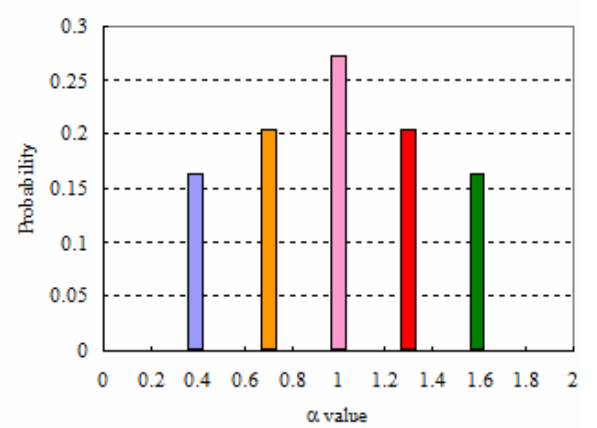

(a) Standard deviation $\sigma_{\mathrm{sd}}=0.389$

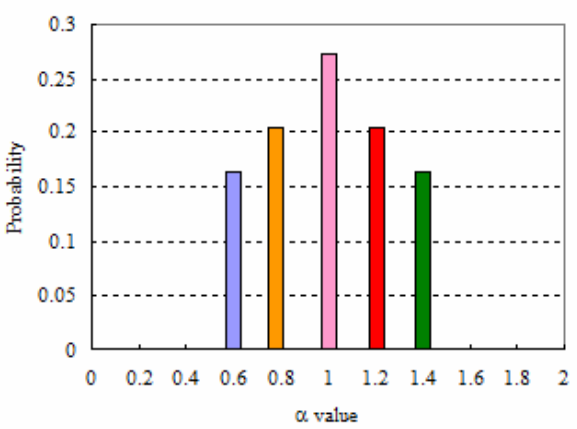

(b) Standard deviation $\sigma_{\mathrm{sd}}=0.260$

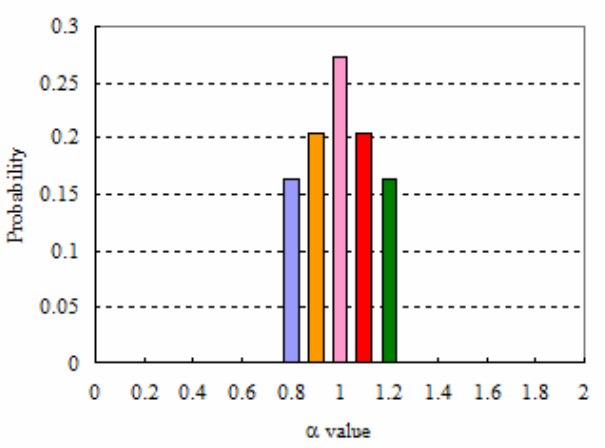

(c) Standard deviation $\sigma_{\mathrm{sd}}=0.130$

Fig. 4 Statistical normal distributions used in the simulation.

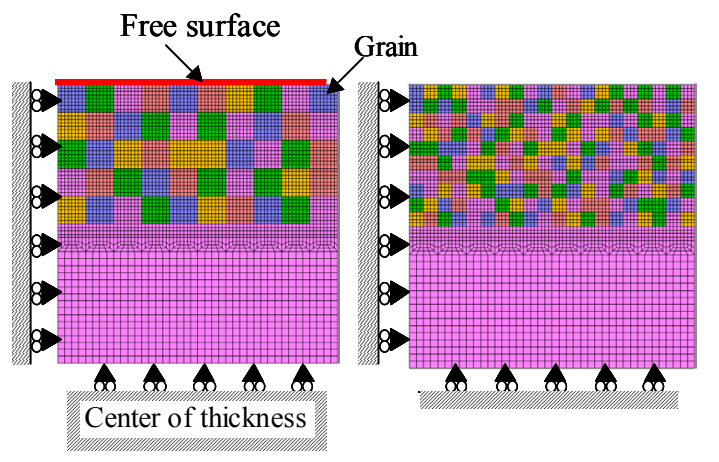

(a) Grain size $d_{g}=25 \mu \mathrm{m}$

(b) Grain size $d_{g}=12.5 \mu \mathrm{m}$

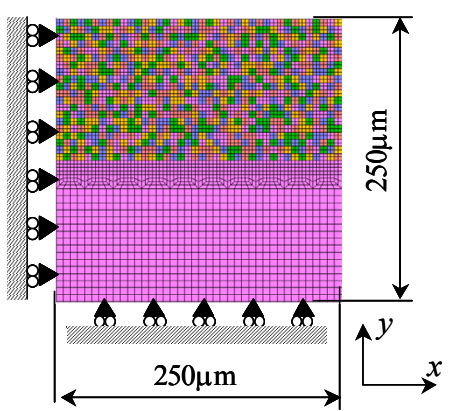

(b) Grain size $d_{g}=6.25 \mu \mathrm{m}$

Fig. 5 Grain sizes used in the simulation under plane strain tensile deformation. 


\subsection{D FE Model}

To verify the proposed model, a 3D FE simulation considering material inhomogeneity was performed. A5052-O aluminum alloy with a thickness of $0.5 \mathrm{~mm}$ was used. A tensile test was conducted to experimentally evaluate fundamental material properties such as flow stress - strain curve, ultimate tensile strength and elongation. A tensile specimen with a gage length of $16 \mathrm{~mm}$ based on a similarly JIS13B specimen was used. The tensile direction was parallel to the rolling direction. The tensile speed was $0.5 \mathrm{~mm} / \mathrm{min}$. Table 1 shows the material properties and grain size of A5052-O. The strength coefficient $K$ and strain hardening index $n$ were determined from the equation $\sigma=K \varepsilon^{n}$. The materials used are considered isotropic macroscopically.

Table 1 Material properties of A5052-O used in experiment.

\begin{tabular}{c|c|c|c|c|c}
\hline Mate rial & $\begin{array}{c}K \text {-value } \\
{[\mathrm{MPa}]}\end{array}$ & $\boldsymbol{n}$-value & $\begin{array}{c}\text { UTS } \\
{[\mathrm{MPa}]}\end{array}$ & $\begin{array}{c}\text { Elongation } \\
{[\%]}\end{array}$ & $\begin{array}{c}\text { Grain size } \\
{[\mu \mathrm{m}]}\end{array}$ \\
\hline A5052-O & 445 & $\mathbf{0 . 3 2}$ & $\mathbf{2 1 1}$ & $\mathbf{2 1}$ & $\mathbf{1 6}$ \\
\hline SPCC & 577 & $\mathbf{0 . 2 3}$ & $\mathbf{3 2 7}$ & $\mathbf{4 6}$ & $\mathbf{1 1}$ \\
\hline
\end{tabular}

To simulate the surface roughening behavior, an FE simulation considering material inhomogeneity was performed. The free surface roughening during plastic deformation was simulated using Marc/Mentat ver.2008. A part of the material is modeled, assuming uni-axial tensile deformation and bi-axial stretch stress state. In this model, crystal grains are assumed to be square with a $20 \mu \mathrm{m}$ side length on the basis of experimental measurements, as shown in Fig. 6. Each element in a group of $4 \times 4 \times 4$ elements has the same material properties. The crystal grains are modeled in the four-layer neighborhood of the free surface because internal crystal grains in the material do not affect the roughening behavior on the free surface ${ }^{(8)}$. The flow stress of internal crystal grains was uniform and the element size of internal crystal grains is larger than that of elements neighboring the free surface to reduce the calculation time. The $\alpha$ values are divided into seven classes. An $\alpha$ value is given to each crystal grain at random on the basis of the normal distribution of probability of $\alpha$. In this material A5052-O, $\alpha$ is assumed to have a standard deviation of $\sigma_{\mathrm{sd}}=0.23(0.55 \leq \alpha \leq 1.45)$ as a result of a fitting process with the results of a tensile test experiment. The $K$ and $n$ values are the average strength coefficients of grains, which means that they represent the microscopic homogeneous strength coefficient. In this study, $K$ and $n$ were obtained from a tensile test. A displacement in the $x$ direction is considered under the tensile condition. The equivalent displacements in the $x$ and $y$ directions are given under the bi-axial stretch stress state.

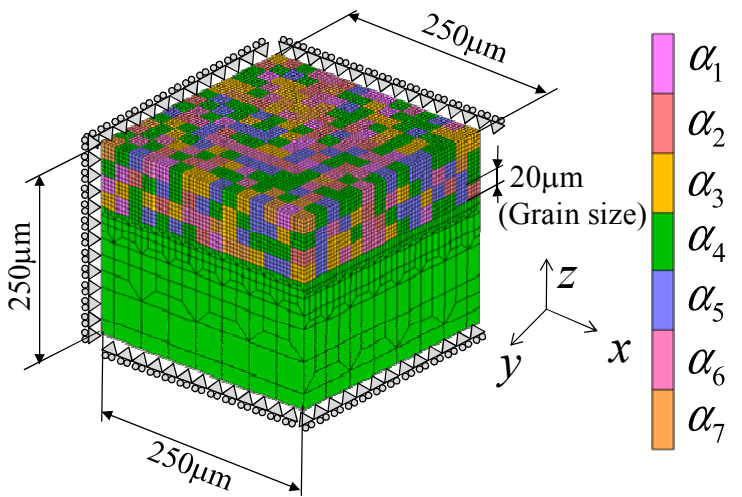

\begin{tabular}{|c|c|c|c|c|c|c|c|c|}
\hline Material & $\alpha_{1}$ & $\alpha_{2}$ & $\alpha_{3}$ & $\alpha_{4}$ & $\alpha_{5}$ & $\alpha_{6}$ & $\alpha_{7}$ & $\begin{array}{c}\text { Standard } \\
\text { deviation } \sigma_{\text {sd }}\end{array}$ \\
\hline$\alpha$ (A5052-O) & 0.55 & 0.70 & 0.85 & 1.00 & 1.15 & 1.30 & 1.45 & 0.23 \\
\hline
\end{tabular}

Fig. 6 3D FE model considering material inhomogeneity for uni-axial and bi-axial tensile deformations. 


\section{Experiments}

To verify the validity of the simulation, a uni-axial tensile test and a bi-axial stretch stress state test were performed and the roughness on the free surface was measured.

Tensile specimens were prepared from the sheets in their rolling direction. In accordance with the JIS standard (Z 2201) tensile specimen 13B, the gauge dimensions was $16 \mathrm{~mm}$. The crosshead speed in the tensile test was set at $0.3 \mathrm{~mm} / \mathrm{min}$. The elongation of the specimen was measured using a noncontact extensometer. The tensile test was halted at each strain. The specimen was removed from the chuck and the surface roughness was measured. At every measurement, care was taken to ensure that the measurement positions were almost the same. Measurements were performed over a travelling length of $3 \mathrm{~mm}$.

The bi-axial tension test was performed by the Marciniak method as shown in Fig. 7. A specimen with blank diameter of $120 \mathrm{~mm}$ was clamped together with a driving plate with a center hole of $17 \mathrm{~mm}$ diameter using an annular blank holder with a head, and was bulged by a flat bottomed punch. At every measurement, care was taken to ensure that those measurement positions were almost the same. Measurements were performed over a travelling length of $3 \mathrm{~mm}$ in the rolling direction.

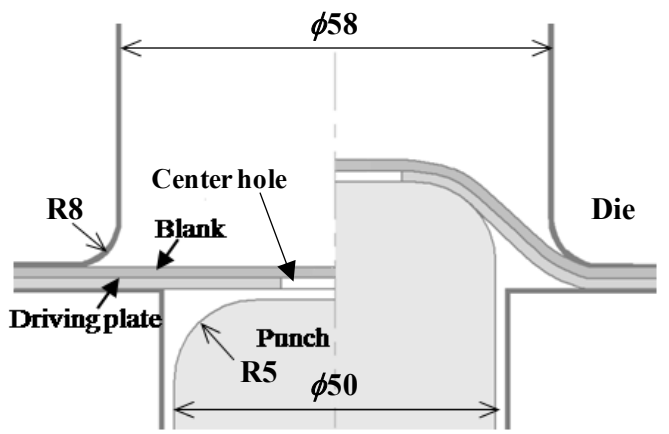

Fig. 7 Schematic illustration of experimental setup for bi-axial stretch test by Marciniak method.

\section{Fundamental Characteristics of 2D Inhomogeneous FE Model}

\subsection{Effect of Material Inhomogeneity Parameter}

The effect of the variation in the material inhomogeneous parameter $\alpha$ on the evolution of roughness on the free surface was investigated. To calculate the surface roughness $R a$, the coordinates of the surface nodes were used. The trace length used for measuring the surface roughness was $250 \mu \mathrm{m}$ in the major strain direction. Figure $\mathbf{8}$ shows the relationship between surface roughness $R a$ and equivalent strain $\varepsilon_{e q}$ for the model with each probability distribution. The rate of increase in surface roughness $R a$ increases with increasing range of variation of $\alpha$ and vice versa. The rate of increase in the surface roughness $R a$ for the model with $0.4 \leq \alpha \leq 1.6\left(\sigma_{\mathrm{sd}}=0.389\right)$ is three-time larger than that for the model with $0.8 \leq \alpha \leq 1.2$ $\left(\sigma_{\mathrm{sd}}=0.130\right)$. This means that the rate of increase in surface roughness is large in the former case because the variation of $\alpha$ is large in case of a large difference in the crystal orientation of each grain. Figure 9 shows the relationship between the standard deviation $\sigma_{\mathrm{sd}}$ of the $\alpha$ value and the rate of increase in the surface roughness. It was found that the standard deviation $\sigma_{\text {sd }}$ of $\alpha$ value has a strong correlation with the rate of increase in the surface roughness.

To verify the validity of this correlation between standard deviation of material inhomogeneity and rate of increase in the surface roughness, the scatter of microscopic hardness was measured experimentally. The average micro Vickers hardness and the variation of hardness value were obtained from the results of one hundred indentation tests 
for Aluminum alloy A5052-O and SPCC. The statistical frequency of the micro Vickers hardness was investigated. Figure 10 shows the statistical distribution of the variation in micro Vickers hardness for A5052-O and SPCC. The hardness histograms for both materials are shaped like a Gaussian distribution. In this study, the dimensionless standard deviation $S_{\mathrm{H}}$ was adopted to ignore the effect of the difference in the average hardness at different materials, and is expressed as,

$$
S_{H}=\sigma_{H V} / \overline{H V}
$$

where, $\sigma_{H V}$ is standard deviation of hardness, $\overline{H V}$ is average hardness, and $S_{\mathrm{H}}$ should be assumed as a parameter of material inhomogeneity of microscopic flow stress. $S_{H}$ and rate of increase in the surface roughness for A5052-O and SPCC was compared. The rate of increase in surface roughness is including the effect of grain size as shown in Eq. (1). Thus, the rate of increase in surface roughness is divided in the average grain size of $16 \mu \mathrm{m}$ for A5052-O and $11 \mu \mathrm{m}$ for SPCC, which expresses $c$ in Eq. (1). Figure 11 shows the relationship between $S_{\mathrm{H}}$ and $c$ for A5052-O and SPCC. It can be seen that $c$ increases with increasing $S_{\mathrm{H}}$. This result cannot be compared with simulation results directly. However, the tendency of the effect of material inhomogeneity on rate of increase in free surface roughness can be demonstrated by comparison of experimental results of hardness tests.

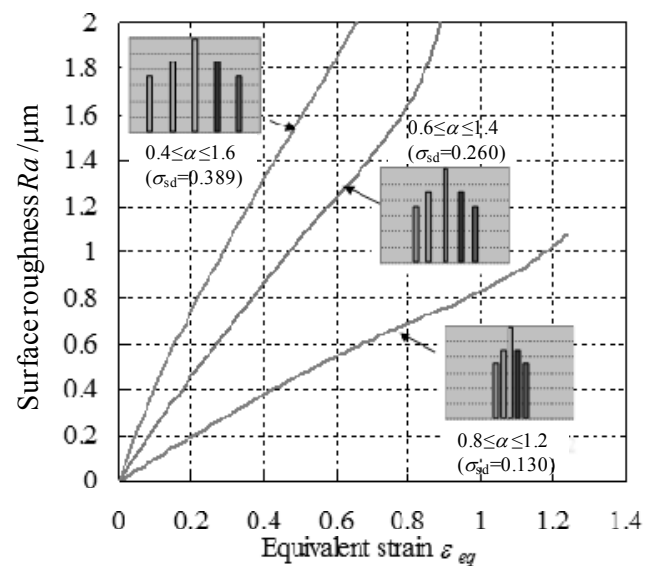

Fig. 8 Effect of standard deviation $\sigma_{\text {sd }}$ of $\alpha$ value on free surface roughening behavior obtained from 2D simulation.

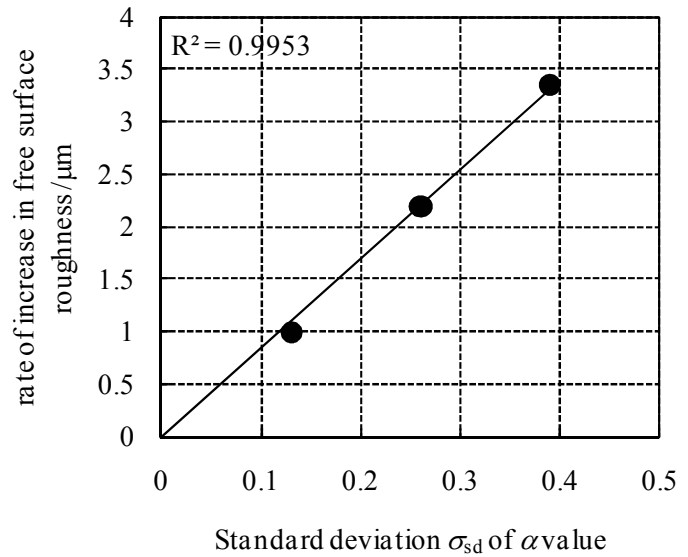

Fig. 9 Relationship between rate of increase in surface roughness and standard deviation $\sigma_{\text {sd }}$ of $\alpha$ value obtained from 2D simulations. 


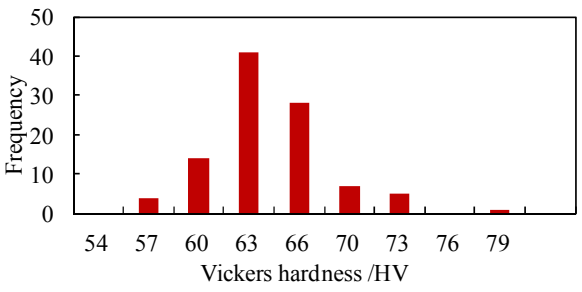

(a) A5052-O

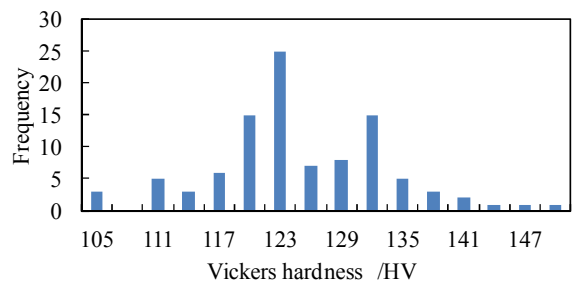

(b) SPCC

Fig. 10 Statistical distribution of variation in micro Vickers hardness for A5052-O and SPCC.

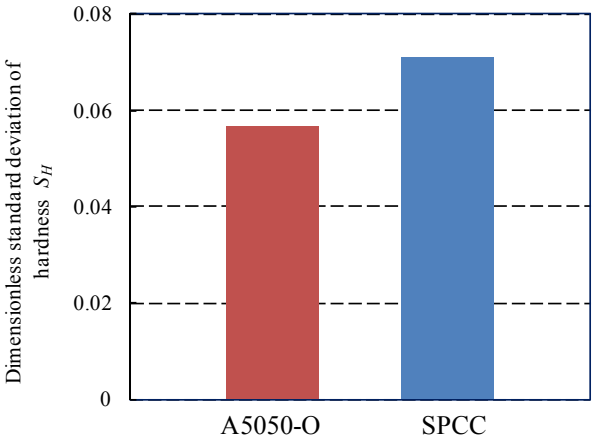

(a) Dimensionless standard deviation of hardness $S_{H}$

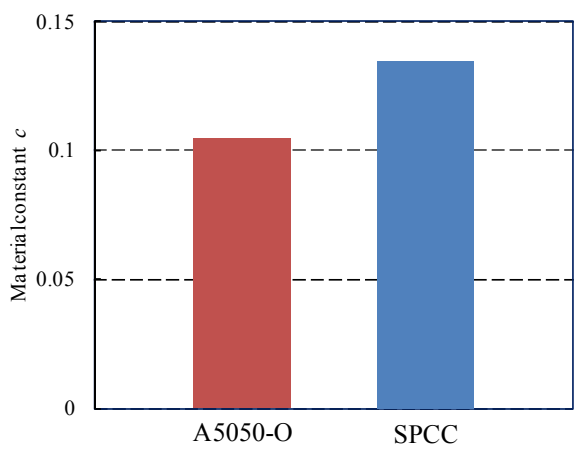

(b) Material constant $c$

Fig. 11 Statistical distribution of variation in micro Vickers hardness for A5052-O and SPCC.

\subsection{Effect of Grain Size}

Figure 12 shows the effect of grain size on the free surface roughening behavior obtained from the 2D FE model. The rate of increase in free surface roughness increases with increasing grain size. Generally, it is known that the gradient of the increase in free surface roughness is dependent on the grain size according to Eq. (1). Thus, the effect of grain size can be modeled in the proposed model considering material inhomogenentiy. Therefore, the validity of the model has been demonstrated for the effect of grain size.

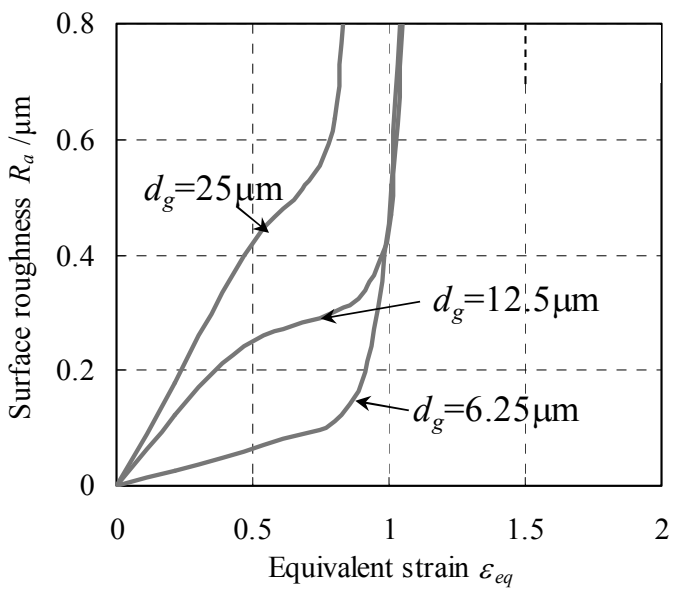

Fig. 12 Effect of grain size on free surface roughening behavior obtained from 2D FE simulations. 
5. Prediction of Free Surface Roughening Behavior by 3D Inhomogeneous FE Model

Figures 13 and 14 respectively shows the free surface roughening behavior after uni-axial tensile and bi-axial tension deformation obtained from the 3D FE simulation considering material inhomogeneity. The free surface roughening, which cannot be expressed in a conventional homogeneous FE simulation, can be represented by the FE simulation considering material inhomogeneity. Surface asperity on the free surface increases with increasing strain. Additionally, directional dependency of the surface roughening cannot be observed from Figures 13 and 14. The surface roughness $R a$ on the free surface during deformation was measured in the FE simulation. Figure $\mathbf{1 5}$ shows the relationship between equivalent strain $\varepsilon_{e q}$ and surface roughness $R a$ during deformation under uni-axial tension state and bi-axial tension. The surface roughness linearly increases with increasing equivalent strain $\varepsilon_{e q}$ in the $\mathrm{FE}$ simulation considering material inhomogeneity. The rates of increase in surface roughness under uni-axial tension and bi-axial tension are the same as those reported in previous studies ${ }^{5-8)}$.

Figure 16 shows the relationship between major strain $\varepsilon_{1}$ and surface roughness $R a$ during deformation under uni-axial tension and bi-axial tension obtained from the FE simulation and experiments. The surface roughness under bi-axial tension is larger than that under uni-axial tension. In addition, the experimental results are in good agreement with the simulation results. Therefore, the validity of the FE model considering material inhomogeneity has been demonstrated.

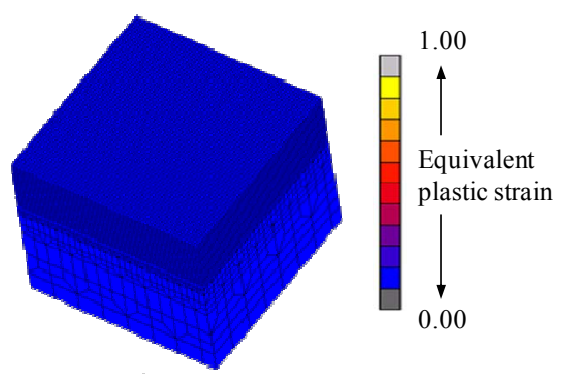

(a) $\varepsilon_{e q}=0$

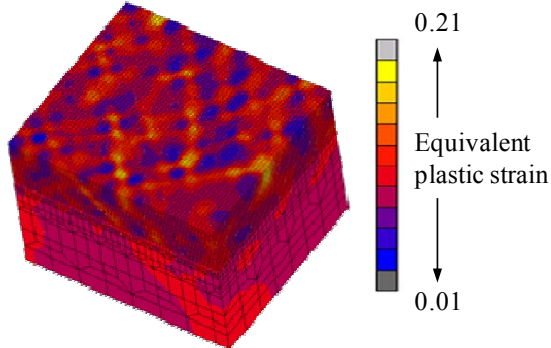

(b) $\varepsilon_{e q}=0.09$

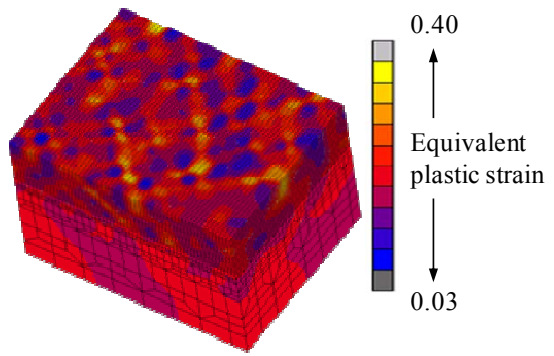

(c) $\varepsilon_{e q}=0.18$

Fig. 13 Free surface roughening behavior during uni-axial tension state. 


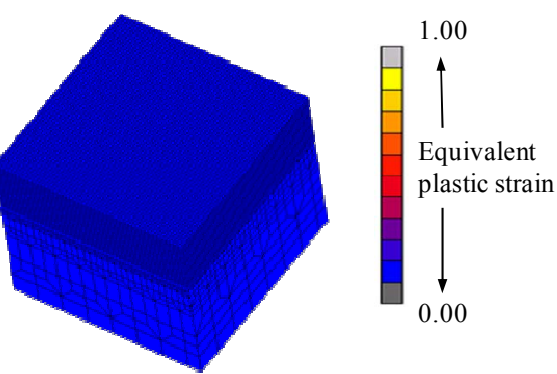

(a) $\varepsilon_{e q}=0$

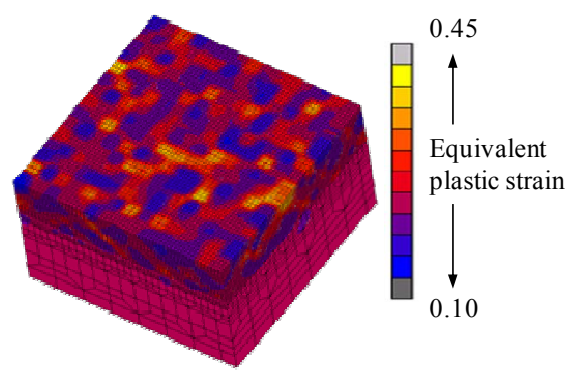

(b) $\varepsilon_{e q}=0.23$

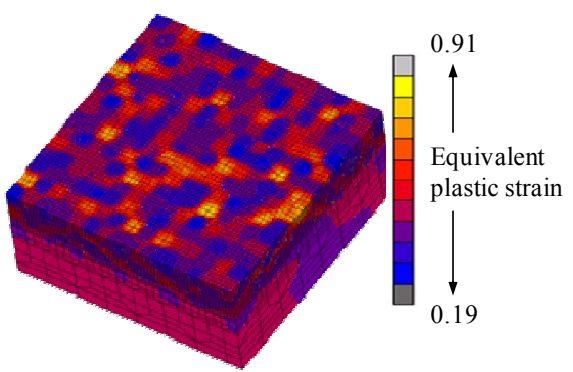

(c) $\varepsilon_{e q}=0.18$

Fig. 14 Free surface roughening behavior during bi-axial tension state.

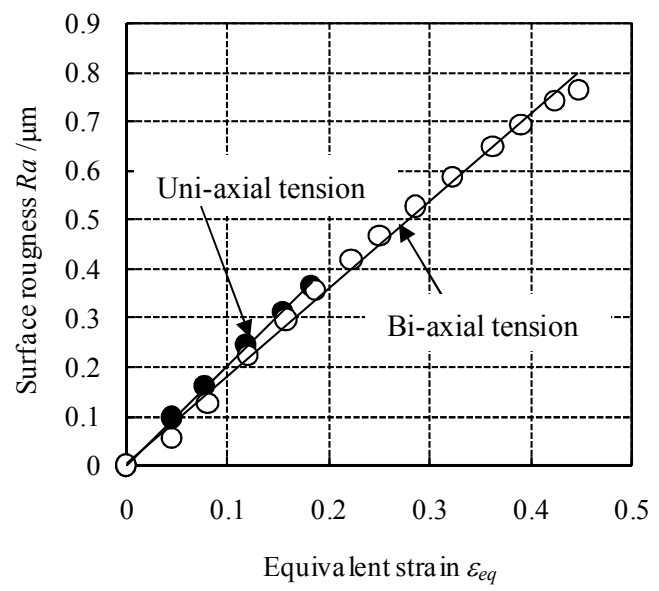

Fig. 15 Relationship between equivalent strain $\varepsilon_{\text {eq }}$ and surface roughness $R a$ during deformation under uni-axial tension and bi-axial tension states.

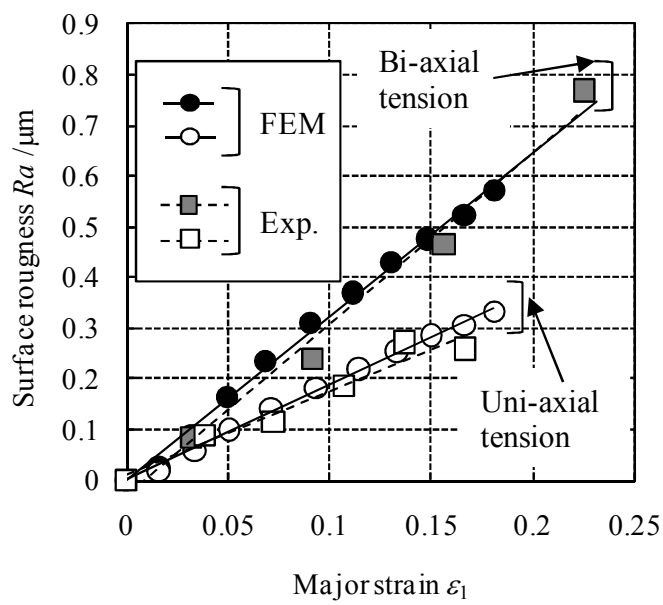

Fig. 16 Relationship between major strain $\varepsilon_{1}$ and surface roughness $R a$ during deformation under uni-axial tensile and bi-axial tension states obtained from 3D FE simulations and experiments. 


\section{Conclusions}

In this study, we proposed an FE model considering mesoscopic material inhomogeneity due to a different flow stress for each crystal grain to predict free surface roughening. The fundamental behavior and validity of this model were verified by $2 \mathrm{D}$ and 3D FE models considering material inhomogeneity.

The following conclusions can be drawn:

(1) The standard deviation $\sigma_{\mathrm{sd}}$ of the material inhomogeneity parameter $\alpha$ in the model has a strong correlation with the rate of increase in the surface roughness.

(2) The rate of increase in the free surface roughness increases with increasing grain size, which is consistent with the conventional empirical equation, Eq. (1).

(3) The surface roughness under bi-axial tension is larger than that under uni-axial tension state. In addition, the experimental results are in good agreement with the simulation results.

From these conclusions, we verified the fundamental behavior and the validity of this model considering material inhomogeneity for the prediction of free surface roughening.

\section{Acknowledgement}

This research was supported by RFBR and JSPS under the Russia-Japan Research Cooperative Program and Grant-in-Aid for Young Scientists (B) by JSPS

\section{Reference}

(1) Engel, U., and Eckstein, R. Microforming-from basic research to its realization, Journal of Materials Processsing Technology, Vol. 125-126, (2002), pp. 35-44.

(2) Saotome, Y., Noguchi, Y., Zhang, T., and Inoue, A., Characteristic Behavior of Pt-based Metallic Glass Under Rapid Heating and its Application to Microforming, Materials Science and Engineering A, Vol. 375-377, (2004), pp. 389-393.

(3) Furushima, T., and Manabe, K., Experimental and Numerical Study on Deformation Behavior in Dieless Drawing Process of Superplastic Microtubes, Journal of Materials Processsing Technology, Vol. 191, (2007), pp. 59-63.

(4) Furushima, T., Noda, Y., and Manabe, K., Laser Dieless Drawing Process for Metal Micro-tubes, Key Engineering Materials, Vol. 443, (2010), pp. 699-704.

(5) Yamaguchi, K., and Mellor, P. B., Thickness and Grain Size Dependence of Limit Strains in Sheet Metal Stretching, International Journal of Mechanical Sciences, Vol. 18, (1976), pp. 85-90.

(6) Osakada, K., and Oyane, M., On the Roughening Phenomenon of Free Surface in Deformation Process, Transaction of the Japan Society of Mechanical Engineers, Vol. 36, (1970), pp. 1017-1022 (in Japanese).

(7) Fukuda, M., Yamaguchi, K., Takakura, N., and Sakano, Y., Roughening Phenomenon on Free Surface of Products in Sheet Metal Forming, Journal of the Japan Society for Technology of Plasticity, Vol. 15, (1974), pp. 994-1002 (in Japanese).

(8) Yamaguchi, K., Takakura, N., and Fukuda, M., FEM simulation of Surface Roughening and Its Effect on Forming Limit in Stretching of Aluminum Sheets, Advanced Technology of Plasticity 1987, (1987), pp. 1267-1274.

(9) Zhao, Z., Radovitzky, R., and Cuitino, A., A Study of Surface Roughening in fcc Metals Using Direct Numerical Simulation, Acta Materialia, Vol. 52, (2004), pp. 5791-5804.

(10)Zhao, Z., Kuchnicki, S., Radovitzky, R., and Cuitino A., Influence of In-grain Mesh Resolution on the Prediction of Deformation Textures in Fcc Polycrystals by Crystal Plasticity FEM, Acta Materialia, Vol. 55, (2007), pp. 2361-2373. 
(11)Utsunomiya, H., Sutcliffe, M.P.F., Shercliff, H.R., Bate, P., and Miller, D.B., Evolution of Matt Surface Topography in Aluminium Pack Rolling. Part I: model development, International Journal of Mechanical Sciences, Vol. 46, (2004), pp. 1349-1364.

(12)Utsunomiya, H., Sutcliffe, M.P.F., Shercliff, H.R., Bate, P., and Miller, D.B., Evolution of Matt Surface Topography in Aluminium Pack Rolling. Part II: effect of material properties, International Journal of Mechanical Sciences, Vol. 46, (2004), pp. 1365-1375.

(13) Furushima, T., Nakata, K., Manabe, K., and Alexansrov, S., Constitutive Modeling of Free Surface Roughening in Sheet Metal Considering Mesoscopic Inhomogeneity Based on Hardness Distribution, Journal of Solid Mechanics and Materials Engineering, Vol. 3, (2009), pp. 1285-1296.

(14) Furushima, T., Manabe, K., and Alexansrov, S., FE Mesoscopic Analysis of Roughness Evolution on Free Surface of Thin Sheet Metals with Strain Rate Sensitivity, Proceedings of the $2^{\text {nd }}$ Asian Workshop on Nano/Micro Forming Technology, (2007), pp. 13-14. 